It cannot be concluded whether the circulatory insufficiency is due to arterial thrombosis or repeated functional obliterations, and whether venous thrombosis precedes arterial circulatory disturbance or not.

\title{
59. Orbito-ventromedial Undercutting: Follow-up Study of 68 Cases, 19571962
}

\author{
Sadao Hirose, Kohzo Odawara and Akira Motegi \\ Dept. of Psychiatry, Nippon Medical College
}

Hirose have employed various methods of psychosurgery on a total of 474 cases since July, 1947. A new method of orbito-ventromedial undercutting was begun in July, 1957. The rise and fall of psychosurgical operations, from my own operative data, are shown in Figure 1.

In the last two reports $(1958,1959)$, at the annual meeting of the Japan Neurosurgical Society, it was maintained by Hirose and Inugami that orbitoventromedial undercutting has been a most effective procedure for the purpose of lessening emotional tention states without undesirable side effects and this

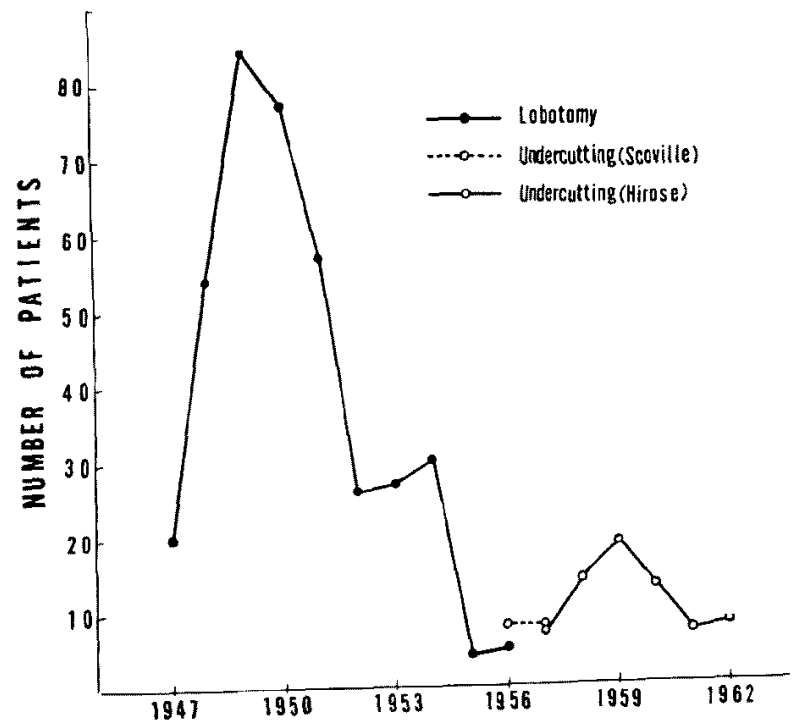

Fig. 1. Rise and fall of prefrontal lobotomy (including transorbital lobotomy), Scoville's selective cortical underculting and Hirose's orbito-ventromedial undercutting. 
method was described in detail.

This is a follow-up study of 68 patients who underwent orbito-ventromedial undercutting for the treatment of mental disorders during the period 1957 to 1962. Four of these patients died in 12 to 40 months after the operation from other disease.

The residential and occupational status of patients is shown in Table 1 and 2. Table 3 and 4 shows the degree of improvement following the operation. Patients operated upon in university hospital fared better than those operated upon in mental hospital. Among the former 25 cases, one to three years after operation, 19 cases $(76 \%)$ are discharged and most of them are either usefully employed or keeping house. Among the latter 39 cases, three to six years after operation, 17 cases $(43.6 \%)$ are discharged. When it is considered that all of these patients had failed to improve under various kind of nonsurgical treatments, the results are impressive.

Marked improvement has been obtained especially in patients with severe

Table 1. Status of Patients Subjected to Orbito-ventromedial Undercutting 1957-1960 - Menta1 Hosp. Cases-

\begin{tabular}{|c|c|c|c|c|c|}
\hline \multirow{2}{*}{ Diagnosis } & \multirow{2}{*}{ Number } & \multicolumn{3}{|c|}{ Discharged } & \multirow{2}{*}{$\begin{array}{l}\text { Insti- } \\
\text { tution }\end{array}$} \\
\hline & & Employed & $\begin{array}{c}\text { Keeping } \\
\text { House }\end{array}$ & Home & \\
\hline Schizophrenia & 19 & 1 & 4 & 1 & 13 \\
\hline Atypical schizophrenia & 2 & 1 & 1 & 0 & 0 \\
\hline Manic depressive psychosis & 1 & 0 & 1 & 0 & 0 \\
\hline Involutional psychosis & 2 & 0 & 1 & 1 & 0 \\
\hline Epilepsy with explosive behavior & 6 & 3 & 1 & 0 & 2 \\
\hline Psychopathic personality (explosive) & 9 & 2 & 0 & 0 & 7 \\
\hline Total & 39 & 7 & 8 & 2 & 22 \\
\hline
\end{tabular}

Table 2. Status of Patients Subjected to Orbito-ventromedial Undercutting 1960-1962 - Univ. Hosp. Cases-

\begin{tabular}{|c|c|c|c|c|c|}
\hline \multirow{2}{*}{ Diagnosis } & \multirow{2}{*}{ Number } & \multicolumn{3}{|c|}{ Discharged } & \multirow{2}{*}{$\begin{array}{l}\text { Insti- } \\
\text { tution }\end{array}$} \\
\hline & & Employed & $\begin{array}{c}\text { Keeping } \\
\text { House }\end{array}$ & Home & \\
\hline Schizophrenia & 9 & 4 & 2 & 1 & 2 \\
\hline Atypical schizophrenia & 4 & 3 & 1 & 0 & 0 \\
\hline Manic depressive psychosis & 1 & 0 & 0 & 0 & 1 \\
\hline Involutional melancholia & 1 & 1 & 0 & 0 & 0 \\
\hline Obsessional neurosis & 3 & 1 & 2 & 0 & 0 \\
\hline Epilepsy with explosive behavior & 5 & 3 & 0 & 1 & 1 \\
\hline Mental deficiency & 2 & 0 & 0 & 0 & 2 \\
\hline Total & 25 & 12 & 5 & 2 & 6 \\
\hline
\end{tabular}


Table 3. Results of Orbito-ventromedial Undercutting Performed 1957-1960 -Mental Hosp. Cases-

\begin{tabular}{l|c||cccc|cc}
\hline Diagnosis & Number & \multicolumn{2}{|c|}{$\begin{array}{l}\text { Symptom- Markedly } \\
\text { free }\end{array}$} & $\begin{array}{c}\text { Moderately } \\
\text { improved improved }\end{array}$ & $\begin{array}{l}\text { Slightly } \\
\text { improved }\end{array}$ & $\begin{array}{l}\text { Unim- } \\
\text { proved }\end{array}$ & Worse \\
\hline $\begin{array}{l}\text { Schizophrenia } \\
\text { Atypical }\end{array}$ & 19 & 0 & 2 & 14 & 3 & 0 & 0 \\
$\begin{array}{c}\text { schizophrenia } \\
\begin{array}{c}\text { Manic depressive } \\
\text { psychosis }\end{array}\end{array}$ & 1 & 1 & 1 & 0 & 0 & 0 & 0 \\
$\begin{array}{c}\text { Involutional } \\
\text { psychosis }\end{array}$ & 2 & 0 & 1 & 0 & 0 & 0 & 0 \\
$\begin{array}{c}\text { Epilepsy with ex- } \\
\text { plosive behavior }\end{array}$ & 6 & 1 & 3 & 2 & 0 & 0 & 0 \\
$\begin{array}{c}\text { Psychopathic } \\
\text { personality } \\
\text { (explosive) }\end{array}$ & 9 & 1 & 2 & 3 & 2 & 1 & 0 \\
\hline Total & 39 & 3 & 10 & 20 & 5 & 0 & 0 \\
\hline
\end{tabular}

Table 4. Results of Orbito-ventromedial Undercutting Performed 1960-1962 -Univ. Hosp. Cases-

\begin{tabular}{|c|c|c|c|c|c|c|c|}
\hline Diagnosis & Number & $\begin{array}{l}\text { Symptom- } \\
\text { free }\end{array}$ & $\begin{array}{l}\text { - Markedly } \\
\text { improved }\end{array}$ & $\begin{array}{l}\text { Moderatel } \\
\text { improved }\end{array}$ & $\begin{array}{l}\text { Slightly } \\
\text { improved }\end{array}$ & $\begin{array}{l}\text { Unim- } \\
\text { proved }\end{array}$ & Worse \\
\hline Schizophrenia & 9 & 0 & 6 & 2 & 1 & 0 & 0 \\
\hline $\begin{array}{l}\text { Atypical } \\
\text { schizophrenia }\end{array}$ & 4 & 1 & 3 & 0 & 0 & 0 & 0 \\
\hline $\begin{array}{l}\text { Manic depressive } \\
\text { psychosis }\end{array}$ & 1 & 0 & 0 & 1 & 0 & 0 & 0 \\
\hline $\begin{array}{l}\text { Involutional } \\
\text { melancholia }\end{array}$ & 1 & 1 & 0 & 0 & 0 & 0 & 0 \\
\hline $\begin{array}{c}\text { Obsessional } \\
\text { neurosis }\end{array}$ & 3 & 1 & 0 & 1 & 1 & 0 & 0 \\
\hline $\begin{array}{l}\text { Epilepsy with ex- } \\
\text { plosive behavior }\end{array}$ & 5 & 0 & 2 & 2 & 1 & 0 & 0 \\
\hline Mental deficiency & 2 & 0 & 0 & 2 & 0 & 0 & 0 \\
\hline Total & 25 & 3 & 11 & 8 & 3 & 0 & 0 \\
\hline
\end{tabular}

involutional melancholia with anxiety, manic depressive psychosis without remission, atypical endogenous psychosis such as recurrent catatonics, schizophrenics with affective coloring, chronic persistent neurosis-like states, and paraphrenia, epilepsy with explosive behavior, and obsessional neurosis. These good results may be partially due to the strict selection of patients for operation, especially in university hospital cases, but is considered largely due to the accurate and effective operative method itself.

Sequels such as confusion, disorientation, amnesia, incontinence, and undesirable personality deficits have never been experienced. There have been no operative deaths. 
Undercutting areas of ventromedial surface as now performed is more restricted (Figure 2) than before (Figure 3).

We aim to elucidate the working mechanism of orbito-ventromedial undercutting on a concrete biological basis such as endocrinologic and electroencephalographic study.

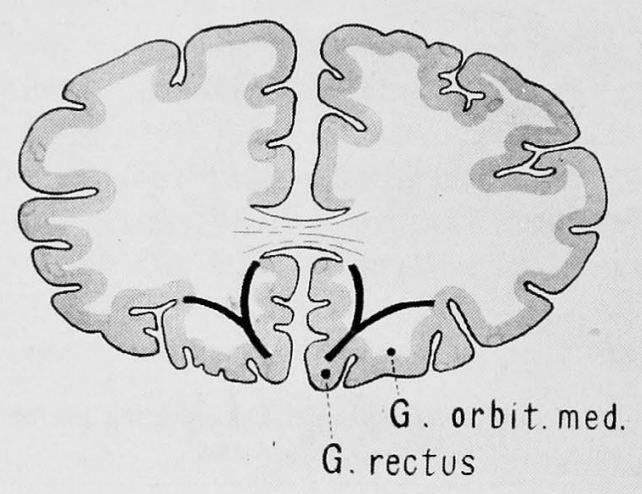

Fig. 2. Orbito-ventromedial undercutting as now performed.

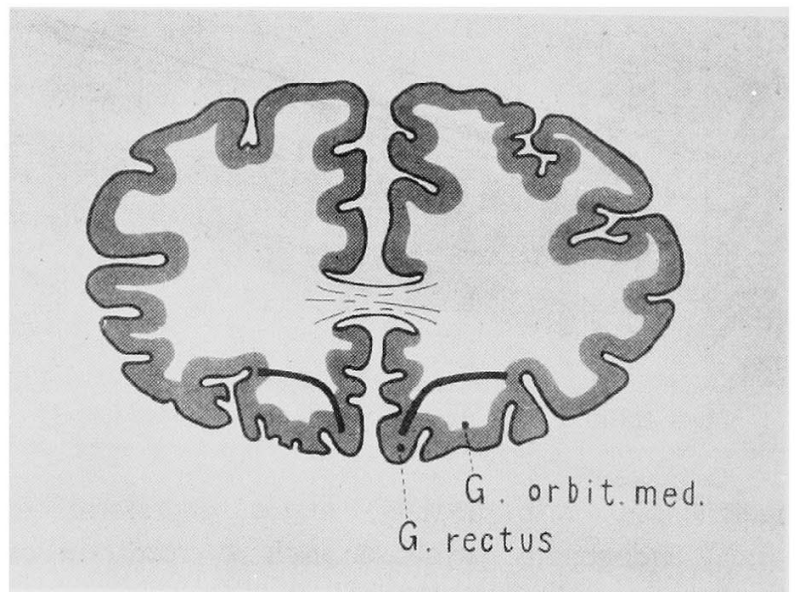

Fig. 3. Orbito-ventromedial undercutting as originally performed. 